\title{
Rheumatoid arthritis and the challenge of using nanoparticles for its treatment
} \author{
Wildan Khairi Muhtadi ${ }^{1}$, and Arif Hidayat ${ }^{4}$ \\ ${ }^{1}$ Faculty of Pharmacy, Gadjah Mada University, Yogyakarta, 55281, Indonesia \\ ${ }^{2}$ Department of Pharmacy, Islamic University of Indonesia, Yogyakarta, 55584, Indonesia \\ ${ }^{3}$ School of Pharmacy Mahaganesha, Denpasar, Bali, 80226, Indonesia \\ ${ }^{4}$ Chemical Engineering Department, Islamic University of Indonesia, Yogyakarta, 55584, Indonesia
}

Lutfi Chabib ${ }^{1,2,{ }^{*}}$, Zullies Ikawati ${ }^{1}$, Ronny Martien ${ }^{1}$, Hilda Ismail $^{1}$, Made Dwi Pradipta Wahyudi $^{3}$, Dewa Ayu Arimurni ${ }^{3}$,

\begin{abstract}
Rheumatoid arthritis is the most common autoimmune disease that affects the joints. The cause of the disease is unknown, many studies proposed hypothesis about the etiology of rheumatoid arthritis. The clinical manifestations of arthritis are different in each patients. In addition, the development of the medication is still continue to achieve the most effective role with less side effect. Nanoparticles may be the answer to this problem, since they have been widely used to improve the pharmacokinetic and pharmacodynamics of rheumatoid arthritis drugs. Using nanoparticles-tagged folate or PEG to deliver rheumatoid arthritis drugs may increase the specificity of the drugs to the target and consequently, may decrease the side effects of the drugs. The purpose of this review is to summarize the etiology, clinical manifestation and highlighting the use of nanoparticles in rheumatoid arthritis treatment.
\end{abstract}

\section{Introduction}

Rheumatoid arthritis is the most common autoimmune disease that affects the joints [1]. This disease associated with progressive disability, systemic complications, early death, and socioeconomic costs [2]. RA is characterised by inflammation of the synovium and increased synovial exudate, which result in thickening of the synovium and joint swelling [3]. The condition is characterised by a symmetrical small joint polyarthritis involving the hands and feet, but larger joints can also be affected. The overall world prevalence of rheumatoid arthritis is approximately $0.5 \%$ to $1 \%$. Based on the data from United Nations World Population Database in 2004, there are over 21 million people worldwide suffering from Rheumatoid arthritis. It can affect people of all age especially in adult in the range 40-50 [4]. The highest incidence appears in Pima indians (5.3\%) and chippewa indians $(6.8 \%)$, and the lowest in people from china and Japan $(0.2 \%-0.3 \%)$ [5]. These finding can be conclude that Rheumatoid Arthritis caused by multifactorial like gene, and environment. The cause of rheumatoid arthritis is still unknown but the leading hypothesis for this disease is the result of an environmental (hormon, dietary factor, infection, ciggarete smoke, and many more) exposure or "trigger" in a genetically susceptible individual [6].

Nowadays, to deliver the drugs to the target site directly is still a problem. Attaching drugs to individually design carriers, like nanoparticles, can make a cellspecific targeting becomes achievable. Many researchers in nanotechnology found that nanoparticles, which exhibit a unique physicochemical and biological property because of their size, is a potential drug carrier.
Nanoparticles have shown that they have the ability to enhance reactive area as well as an ability to cross cell and tissue barriers. That will be the reason behind the widely usage of nanoparticles for biomedical applications [7].

\section{Rheumatoid arthritis}

\subsection{Etiology}

Even the cause of rheumatoid arthritis is still unknown, however many studies have explained that rheumatoid arthrtitis is a result from the interaction of genetics, environment, and the immune system [8].

\subsection{Genetic}

More than $80 \%$ of patients carry the epitope ofthe HLADRB $1 * 04$ cluster [9]. Patients expressing two HLADRB $1 * 04$ alleles have a greater effect on gain that lead to nodular disease, major organ involvement and surgery related to joint destruction [10]. Another gen that have role in RA is A1-B8-DR3 which is the haplotype located near the haplotype and HLA-DPB1 gene [1]. Other RAassociated loci are PTPN22, PADI4, STAT4, TRAF1-C5 and TNFAIP3. The various gen above are MHC family, but non-MHC also can lead to this disease only 3-5\% possibility. Environmental factors, such as smoking and infection, may also interfere the development, rate of progression and severity of RA [11].

\footnotetext{
* Corresponding author: lutfi.chabib@uii.ac.id
} 


\subsection{Environment}

Multiple environmental factors including hormones, dietary factors, infections and exposure tobacco smoke as well as associated with increased risk for rheumatoid arthritis (RA) [12].

\subsubsection{Smoking}

Multiple case-control and prospective cohort studies have demonstrated that cigarette smoking is the strongest environmental factor linked with RA, and it can increased the risk of rheumatoid arthritis until 25\% [13]. Furthermore, several studies demonstrate a dose-response between heavier smoking and RA, some studies show that people who have the habit of smoking more than 20 years led to persistent RA [14]. However, the specific mechanism of how smoking can induce autoimmunity is still unknown.

\subsubsection{Reproductive and hormon}

The abundant study evidence shows there is relation between hormon and incident of RA. Women are 2 to 4 times more likely than men to develop RA [15]. women with irregular menstruation and menarche under 10 years, may increase the risk of RA [16].

\subsubsection{Infection}

The body's immune system consists of cellular and humoral. When there is an infection, the immune system raises inflammatory substances to eliminate infectious agents, this inflammation can occur in the joints. The best example to explain this concept is an infection of porphyromonas gingivalis. The causal direction remains uncertain, but recent discoveries suggest that inflammation and in particular infection by Porphyromonas gingivalis maybe important in the pathophysiology of RA development [17]. The current hypothesis is that Porphyromonas gingivalis mediated citrullination of human peptides might responsible for the initial breakdown in self-tolerance that leads to the development of RA-related autoimmunity [18].

\subsubsection{Dietary intake}

The type of food or nutrient which is having relation with RA are vitamin D and protein. Study show that vitamin D has pleotropic effects on the immune system, inhibiting pro-inflammatory cytokines, upregulating antiinflammatory cytokines, and regulating the innate and adaptive immune system through the vitamin $\mathrm{D}$ receptor [19]. In addition, Vitamin D prevents the development of inflammatory arthritis in collagen-induced mouse models [20]. Foods contain high protein and $\mathrm{Fe}$ is red meat. Based on the study of Norfolk Arthritis Register (NOAR, Great Britain) found the relationship between protein from red meat with incident RA [21].

\subsection{Clinical manifestation}

Symptoms appear in patients with RA vary, according to Arthritis Research UK, the common symtomps are joint pain (three or more joint) and swelling, morning stiffnes (at least one hour), anemia flu-like symtomps (such as feeling generaly ill, feeling hot and sweating), fatigue, irritability, and depression [4]. Another less common symtomp are weight loss, eye inflamation (Episcleritis), rheumatoid nodule, inflamation in other body part such as lung, blood vessel (Vasculitis), entrapment neuropathies median nerve involvement sjogren's syndrome(deficient moisture production of the lacrimal, salivary and otherglands), lymphadenopathy (enlargement of the lymph nodes or vessels), and less common symtomp are felty's syndrome (hypersplenism) and pericarditis [4].

\section{Therapeutic drug for rheumatoid arthritis}

The goal of acute onset therapy for rheumatoid arthritis is to control the underlying inflammatory disease [22]. Attainment of this goal will alleviate pain, improving patients' quality of life, and preserve independence and ability to perform activities of daily living and vocational and avocational pursuits. Furthermore, major long-term goals of treatment are to prevent joint destruction and prevent comorbidities of disease and treatment, including heart disease and osteoporosis [23].

There are four main groups of drugs are used to treat RA. These are painkillers (analgesics), non steroidal antiinflamatory drugs (NSAIDs), disease-modifying antirheumatic drugs (DMARDs), and steroids [4]. RA patients generally consume more than one type of drug, this is due to every drug has different mechanisms and effects.

\subsection{Analgesics}

As explained earlier, that one of the major symptoms of RA are joint pain. This condition will really disrupt the patients, because the joint is the main organ of the movement. Analgesics works as an painkillers. Among analgesics commonly used are type of opioid, paracetamol and aspirin, the other is co-codamol, coproxamol, co-drydramol and tylex. Some analgesics containing codeine can cause constipation [4].

\subsection{Steroid}

Some steroid or corticosteroid produced in the body, for example cortisol. Currently, steroids are widely produced and used to reduce pain. This medication can be used intravenously or orally. As for the side effects can result from steroid medications are facial flushing, interference in menstrual cycle, thinning, and other change in the site of injection (atrophy). But oral tablet has more side effects including weight gain, osteoporosis, muscle weakness, cataract, a rise of blood sugar or blood pleasure, and increased of developing infections [4]. 


\subsection{Non steroidal anti-inflamatory drugs (NSAIDs)}

NSAID is used to relieve pain and swelling. NSAID works by inhibiting the enzyme siklooksigenase. There are two COX isoforms and non-selective NSAIDs inhibit both COX-1 and COX-2 in differing ratios. Some of NSAID are ibuprofen dan diclofenac sodium, cox II selective, meloxicam, Cox II specific, celecoxib. Opioids is also used to relieve moderate to severe pain, some of them are dihydrocodeine tartrate, tramadol hydrochloride. The form of NSAID commonly tablet, capsules, dan liquid. In addition NSAIDs can also be given as a patch or cream that can be used directly at the site of action. but the use of NSAIDs can cause many side effects depends on the dose and duration of therapy, the most severe is the gastrointestinal (GI) and cardiovascular side effects are of particular concern [24].

\subsection{Disease-modifying anti-rheumatic drugs (DMARDs)}

DMARDs mecanism are to reduce pain, swelling and stiffness over a period of weeks or months by slowing down the disease and its effects [4]. This drug is contraindicated for patients with moderate-to-severe heart failure, active tuberculosis. the DMARDs for use in RA include ciclosporin A, hydroxycholoroquine (HCQ), leflunomide (LEF), methotrexate (MTX), intramuscular gold, penicillamine and sulfasalazine (SASP). The efficacy of MTX, intramuscular gold, LEF, penicillamine and SASP, is similar. Intramuscular gold has the highest toxicity and therefore increased treatment drop-out rates compared to SASP, HCQ and MTX. Systematic review found LEF, MTX and SASP to have comparable efficacy. MTX has the most favourable efficacy/toxicity trade-off. SASP scored close to MTX and had more adverse events initially. HCQ had a relatively low rate of toxicity. Essentially, these are monoclonal antibodies (MAbs) directed against chemical mediators of inflammation,notably TNF-a, and interleukins IL-1 and IL-6. These MAbs compete withthe endogenous ligands at their receptor sites on cells, e.g. CD20 and CD22, some of which have no identified endogenous ligand [24].

\section{Challenge}

Commonly, RA was treated symptomatically with nonsteroidal and steroidal anti-inflammatory drugs. However, use of these drugs encountered many obstacles. The most corrosive effects of nonsteroidal drugs such as aspirin on the gut and the serious effects of steroidal drugs is water retention and redistribution of body fat. To reduce these effect is used paracetamol, but it has a relatively low therapeutic index. Furthermore these treatments do not slow the progression of tissue damage and loss mobility. Several drugs, e.g. methotrexate, penicillamine, gold and azathioprine, commonly known as DMARDs, or diseasemodifying anti-rheumatic drugs, have been used for several years. These may provide symptomatic relief and slow the progression of the disease, but are associated with serious adverse effects and relatively non-specific in their actions. The harmful effect is that MAbs suppress the immune response and may facilitate opportunistic infections. Therefore it is challenge that the other drug therapy effective in inhibiting the progression of RA but fewer side effects.

\section{New molecules for rheumatoid arthritis}

Recently it has been proved that biologic RA therapy can solve the problem, this substance works specifically targeted. The target specific therapies either act on the cytokines or its receptors; thus modify immune mediated damage during the "window of opportunity". The potential targets in RA include cytokines such as TNFa, IL1 and IL6, B cells, molecules that cause interaction between antigen presenting cells and $\mathrm{T}$ cells, RANK ligand (receptor activator of nuclear factor $\mathrm{kB}$ ligand), and small molecules that mediate intracellular signalling. $\mathrm{TNFa}$, which is produced by activated macrophages, monocytes, fibroblasts, mast cells, and natural killer (NK) cells, is one of the main cytokines that plays a significant role in the pathophysiology of RA. TNFa possesses an ability to induce signaling pathways of the immune system that lead to the tissue inflammation [25]. Currently, There are nine biologics approved for the treatment of RA, including TNF inhibitors (infliximab, etanercept, adalimumab, certolizumabpegol, and golimumab), anakinra (IL-1 receptorantagonist), abatacept (CTLA4-Ig fusion protein), rituximab (antiCD20 antibody), and tocilizumab (anti-IL-6 receptor antibody). Rituximab and tocilizumab are currently approved for RA patients who have failed treatment with at least $1 \mathrm{TNF}$ inhibitor. Denosumab is amonoclonal antibody against RANKL which is playing role in the differentiation of macrophages to osteoclasts as well as activation of osteoclasts to cause rheumatoid bone erosions. Blocking the action of RANKL with denosumab is therefore likely to be useful in preventing erosions. The type of biological molecule which is currently widely observed by researcher is kinase inhibitor (janus kinases or JAKs, spleen tyrosine kinase or SyK, and p38 mitogenactivated protein kinases or MAPK), which targeted intracellular molecules involved in signal transduction, are also currently in development. Other new targets are regulatory $\mathrm{T}$ cell activation agonist, granulocyte colonys timulating factor (GCSF) and granulocyte macrophage colony-stimulating factor (GMCSF) antagonists than can significantly role in early stage of development of RA [26]. In Addition, nowadays being develop nanoparticle delivery system, which can be combined with antiinflamation natural product from curcumin to eradicate inflamation in RA. This product is expected to be the best solution for RA treatment because the product is biodegradable with high bioavailibility [27].

\section{Nanoparticle challenge as novel treatment of rheumatoid arthritis}

Nanoparticles delivery system has been describe as particles formulation which been disperse in the scale of 
nanometer. NNI (National Nanotechnology Iniative) defined nanoparticles as structures of sizes ranging from 1 to $100 \mathrm{~nm}$. This size can be easily characterize by visual observation. Usually, they have a slightly transparent appearance and have a lower precipitating time compare to another particle with size more than $100 \mathrm{~nm}$ [28].

There are 2 types of nanoparticles based upon their preparation, such as nanospheres and nanocapsules [29]. Nanospheres has a monolithic type structure, where the active compound is disperse actively in the surface or adsorbed into the surface of its carrier matrix. Nanocapsules form a membrane-like structure with the active compound trapped in to the "heart" of its structure or adsorbed on the membrane surface. However, it is hard to classify a nanoparticles complex in to nanospheres or nanocapsules type [30].

One of the simplest preparation methods of polymeric nanoparticles is via ionic gelation method. Ionic gelation concept make the usage of two biopolymers in one formulation system becomes possible. The two biopolymers should have a different charge, so they can form a flexible matrix to entrap various kind of drugs with different characteristics. For example, many researchers used a combination of chitosan-alginate [31] or chitosancarrageenan [32] that can be used to physically entrap drugs. Grenha [32] reported that chitosan-carrageenan nanoparticles complex could become a carrier for ovalbumin with entrapment efficiency around $4-17 \%$. On another research, Gazori [33] used a combination of chitosan-alginate to make nanoparticles. They reported that this combination could increase drug stability in the systemic fluid.

Some of the advantages of using nanoparticles are their small size enable them to penetrate intercellular spaces [34], cell walls compared to bigger particles, and flexibility to combine with other technologies [35]. Nanoparticles can be used to encapsulate, protect drugs from degradation, and improve targeted drug delivery. Nanoparticles can modify the drug release, and can be produce in large, reproducible scale [36]. Nanoparticles' wide variety of physical and chemical properties affect the encapsulated drug biomedical potential such as bioavailability and biodistribution [37]. The use of nanomaterials particulate as drug carrier, resulted accumulation of nanoparticles in mononuclear phagocytic system that present in spleen and liver, therefore liver targeting by nanoparticle may be favorable and could be a promising drug therapy for liver diseases. Surface modification of nanoparticles particularly with PEG, could prolong its residence time in the circulation, reduce in vitro toxicity, prevents agglomeration that lead to destabilization of nanoparticle suspension [38]. The other advantage is that the drugs in the form of nanoparticles possess good storage stability. The study examined the storage stability of self-nanoemulsifying drug delivery systems (SNEDDS), which one of the nanoparticle based drug deliveries, found that the SNEDDS indicated no phase separation and no significant changes on the particle size were found during several tests that had been conducted. The small particle size of the nanoparticle based dosage form prevents creaming or sedimentation due to a decrease of gravitation and Brownian motion.
The small particle size also prevents the flocculation to occur, which allows the drugs to be stored for a long period [39].

Despite various benefits of nanoparticle as drug carrier, there are several disadvantages such as the possibility to generate toxicity. Due to small size of nanoparticle, it has wide biodistribution in the body and increase the potency of drug, which not only beneficial for therapy but also increase the possibilities of adverse effect of attached drug in the organs other than the main target of therapy. Nanoparticles also known to affect immune response to common allergens.

Nanoparticles have been widely used to improve the pharmacokinetic and pharmacodynamics of drug molecules. Most of the non-steroidal anti-inflammatory drugs that commonly used to treat RA have a low solubility in gastric fluid but have a good solubility in intestine fluid [40]. Many researchers have proved the ability of nanoparticles in improving the bioavailability of drugs with low solubility [41] while delivered via oral [42], transdermal [43], intravenous [44], and pulmonary $[45,46]$. On the other hand, the improvement of the amount of drugs in systemic will increase the risk of side effects occurrence until the possibility of achieving toxic level [47]. The compatibility between nanoparticles dosage form and target tissue is needed to achieve an optimum therapy results. The guarantee of target therapy achievement becomes an absolute requirement to introduce a new drug delivery system.

Many researchers have been proposed so many materials to deliver drugs for treating cancer. Those materials have been used to deliver rheumatoid arthritis therapeutic agents in recent years. Nanoparticles, like micelles and liposome, can be further improved its specificity and therapeutic efficacy by combining with an antibody, peptide, or polysaccharide whose specific for the receptors located within tissue affected by RA. By combining passive and active targeting strategies, the delivery of drugs can be optimized, so the toxicity and the unwanted side effect will be reduce, and in the end it will improve patient outcome [48].

Nanoparticle delivery systems of RA drugs primarily based upon polymers. For example, many researches have been done to explore the use of poly(D,L-lactic/glycolic acid) (PLGA) nanoparticles. The PLGA nanoparticles shows a promising result that it can prolong the circulation time and control the drug release from matrix [48]. Higaki [49] make a betamethasone nanoparticles using PLGA with the size of particles around 100-200 nm. On that research, they found that the PLGAbetamethasone system was more effective to reduce the inflammatory response on arthritic rats and mice while administered intravenously compare to free glucocorticoid agents. The modification of PLGA nanoparticles with PEG can improve the targeting ability and efficacy of betamethasone. That system is called "stealth nanosteroids" [50].

Nowadays, the development of drug delivery systems for rheumatoid arthritis treatment is based upon the principles of passive targeting. The improvement of drugs specificity can be achieved by incorporating an active targeting compound like folate receptor $\beta$, which is an 
active targeting compound of Rheumatoid Arthritis Synovial Macrophage (RASMs). The researches show that the system of cationic and anionic poly(amidoamine) dendrimers loaded with indomethacin exhibit better effect in treating arthritic rats when incorporated with folate [5153]. Therefore, the combination of nanoparticles with folate or with folate-PEG may be potential to be used as drug delivery system for RA treatment in the future.

\section{Conclusion}

Rheumatoid arthritis is the most common autoimmune disease that Affects the joints. RA is characterized by inflammation of the synovium and synovial Increased exudate which result in thickening of the synovium and joint swelling. Cause of RA is unknown, but the most stronger hypothesis is as a result of the interaction between genes and the environment. The drugs used for RA are steroids, NSAIDs, and DMARDs. However the drugs effectiveness is still scanty and harmful side effects. Nowadays researchers are being developed RA drugs that tend to inhibit the progression of RA in early development. It is still a challenge for researchers to find precise formula which is effective and have minimal side effects. Thus, incorporating drugs in nanoparticles-tagged folate or PEG may become a promising delivery system for rheumatoid arthritis treatment.

We would like to acknowledge Curcumin Research Centre, Faculty of Pharmacy, Gadjah Mada University and Pharmacy Nanoscience, Pharmaceutical Technology Laboratory, Department of Pharmacy, Islamic University of Indonesia. We also would like to address a gratitude to the grant of Penelitian Disertasi Doktor Kemeristekdikti. Authors declare no conflict of interest.

\section{References}

1. I. B. McInnes, G. Schett, The Pathogenesis of Rheumatoid Arthritis, N Engl J Med, 365, 22052219, (2011).

2. G. S. Firestein, Evolving concepts of rheumatoid arthritis, Nature, 423, 356-361, (2003).

3. S. Ryan, Continuing professional development, autoimmune disease, Royal College of Nursing, 16, (2002).

4. UK AR, Condition Rheumatoid Arthritis United Kingdom, Arthritis Research UK, (2014).

5. A. Gibofsky, Overview of epidemiology, pathophysiology, and diagnosis of rheumatoid arthritis, Am J Manag Care, 18, 295-302, (2012).

6. A. Gibofsky, R. J. Winchester, M. Patarroyo, M. Fotino, H. G. Kunkel. Disease associations of the Ialike human alloantigens: contrasting patterns in rheumatoid arthritis and systemic lupus erythematosus, J Exp Med, 148, 1728-1732, (1978).

7. A. Z. Wilczewska, K. Niemirowicz, K. H. Markiewicz, H. Car, Nanoparticles as drug delivery systems, Pharmacol Rep, 64, 1020-1037, (2012).
8. D. S. Majka, K. D. Deane, L. A. Parrish, A. A. Lazar, A. E. Baron, C. W. Walker, M. V. Rubertone, W. R. Gilliland, J. M. Norris, V. M. Holers, The duration of preclinical rheumatoid arthritis-relatedautoantibody positivity increases in subjects with older age at time of disease diagnosis, Ann Rheum Dis, 67, 801-807, (2008).

9. J. S. Smolen, G. Steiner, Therapeutic strategies for rheumatoid arthritis, Nat Rev Drug Discov, 2, 473488, (2003).

10. J. S. Smolen, D. Aletaha, M. Koeller, M. H. Weisman, P. Emery, New therapies for treatment of rheumatoid arthritis. Lancet, 370, 1861-1874, (2007).

11. M. T. Getts, S. D. Miller, 99th Dahlem conference on infection, inflammation and chronic inflammatory disorders: triggering of autoimmune diseases by infections, Clin Exp Immunol, 160, 15-21, (2010).

12. E. W. Karlson, K. Deane, Environmental and geneenvironment interactions and risk of rheumatoid arthritis. Rheum Dis Clin North Am, 38, 405-426, (2012).

13. K. H. Costenbader, D. Feskanich, L. A. Mandl, E. W. Karlson, Smoking intensity, duration, and cessation, and the risk of rheumatoid arthritis in women, Am J Med, 119, 503-511, (2006).

14. L. Klareskog, P. Stolt, K. Lundberg, H. Källberg, C. Bengtsson, J. Grunewald, J. Rönnelid, H. E. Harris, A. K. Ulfgren, S. Rantapää-Dahlqvist, A. Eklund, L. Padyukov, L. Alfredsson, A new model for an etiology of rheumatoid arthritis: Smoking may trigger HLA-DR (shared epitope)-restricted immune reactions to autoantigens modified by citrullination, Arthritis Rheum, 54, 38-46, (2006).

15. D. P. Symmons, E. M. Barrett, C. R. Bankhead, D. G. Scott, A. J. Silman, The incidence of rheumatoid arthritis in the United Kingdom: results from the Norfolk Arthritis Register, Br J Rheumatol, 33, 735739, (1994).

16. E. W. Karlson, L. A. Mandl, S. E. Hankinson, F. Grodstein, Do breast-feeding and other reproductive factors influence future risk of rheumatoid arthritis? Results from the Nurses' Health Study, Arthritis Rheum, 50, 3458-3467, (2004).

17. K. Lundberg, N. Wegner, T. Yucel-Lindberg, P. J. Venables, Periodontitis in RA-the citrullinated enolase connection, Nature Reviews Rheumatology, 6, 727-730, (2010).

18. N. Wegner, R. Wait, A. Sroka, S. Eick, K. A. Nguyen, K. Lundberg, A. Kinloch, S. Culshaw, J. Potempa, P. J. Venables, Peptidylarginine deiminase from Porphyromonas gingivalis citrullinates human fibrinogen and alpha-enolase: implications for autoimmunity in rheumatoid arthritis, Arthritis Rheum, 62, 2662-2672, (2010).

19. Y. Arnson, H. Amital, Y. Shoenfeld, Vitamin D and autoimmunity: new aetiological and therapeutic considerations, Ann Rheum Dis, 66, 1137-1142, (2007). 
20. M. T. Cantorna, C. E. Hayes, H. F. DeLuca, 1,25Dihydroxycholecalciferol inhibits the progression of arthritis in murine models of human arthritis, J Nutr, 128, 68-72, (1998).

21. D. J. Pattison, D. P. Symmons, M. Lunt, A. Welch, R. Luben, S. A. Bingham, K. T. Khaw, N. E. Day, A. J. Silman, Dietary risk factors for the development of inflammatory polyarthritis: evidence for a role of high level of red meat consumption, Arthritis Rheum, 50, 3804-3812, (2004).

22. J. M. Davis, E. L. Matteson, My treatment approach to rheumatoid arthritis, Mayo Clin Proc, 87, 659-673, (2012).

23. L. F. Callahan, D. S. Cordray, G. Wells, T. Pincus, Formal education and five-year mortality in rheumatoid arthritis: mediation by helplessness scale score, Arthritis Care Res, 9, 463-472, (1996).

24. SIGN (Scottish Intercollegiate Guidelines Network), Management of early rheumatoid arthritis, Edinburgh, (2011). (SIGN publication no. 123). [cited February 2011]. Available from URL: http://www.sign.ac.uk

25. R. Awaluddin, W. K. Muhtadi, L. Chabib, Z. Ikawati, R. Martien, H. Ismail, Molecular docking and ADME-toxicity studies of potential compounds of medicinal plants grown in Indonesia as an antirheumatoid arthritis, AIP Conf Proc, 1823, 020033, 1-9, (2017).

26. M. Joshi, Advances in the management of rheumatoid arthritis, J Mahatma Gandhi Inst Med Sci, 17, 1-7, (2012).

27. L. Chabib, Z. Ikawati, R. Martien, H. Ismail, Review of rheumatoid arthritis: the pharmacological therapy, the potential of curcumin and its analogues, and the development of nanoparticle system, Jurnal Pharmascience, 3, 10-18, (2016).

28. R. B. Gupta, U. B. Kompella, Nanoparticle technology of drug delivery, Taylor \& Francis Grup, New York, 4-6, 13-16, (2006).

29. E. Alleman, R. Gurny, E. Doelker, Drug-loaded nanoparticles-preparation methods and drug targeting issues, Eur. J. Pharm. Biopharm., 39, 173-191, (1993).

30. W. Tiyaboonchai, Chitosan nanoparticles: A promising system for drug delivery, Naresuan Univ. J., 11, 51-66, (2003).

31. P. Li, Y. Dai, J. Zhang, A. Wang, Q. Wei, Chitosanalginate nanoparticles as a novel drug delivery system for nifedipine, Int J Biomed Sci, 4, 221-228, (2008).

32. A. Grenha, M. E. Gomes, M. Rodrigues, V. E. Santo, J. F. Mano, N. M. Neves, R. L. Reis RL, Development of new chitosan/carrageenan nanoparticles for drug delivery application, J Biomed Mater Res, 92, 12651272, (2009).

33. T. Gazori, M. R. Khoshayand, E. Azizi, P. Yazdizade, A. Nomani, I. Haririan, Evaluation of alginate/chitosan nanoparticles as antisense delivery vector: formulation, optimization and in vitro characterization, Carb. Pol., 77, 599-606, (2009).

34. C. Buzea, L. Pacheco, K. Robbie, Nanomaterials and nanoparticles: sources and toxicity, Biointerphases, 2, 17-71, (2007).

35. Y. Kawashima, H. Yamamoto, H. Takeuchi, Y. Kuno, Mucoadhesive DL-lactide/glycolide copolymer nanoparticles coated with chitosan to improve oral delivery of elcatonin, Pharm Dev Technol, 5, 77-85, (2000).

36. A. C. Anselmo, S. Mitragotri, Cell-mediated delivery of nanoparticles: taking advantage of circulatory cells to target nanoparticles, J Control Release, 190, 531541, (2014).

37. R. Stevenson, A. J. Hueber, A. Hutton, I. B. McInnes, D. Graham, Nanoparticles and inflammation, Sci World J, 11, 1300-1312, (2011)

38. T. Niidome, M. Yamagata, Y. Okamoto, Y. Akiyama, H. Takahashi, T. Kawano, Y. Katayama, Y. Niidome, PEG-modified gold nanorods with stealth character for in vivo application, J Control Release, 114, 343347, (2006)

39. L. Chabib, W. K. Muhtadi, Z. Ikawati, R. Martien, H. Ismail, Stability study of gamavuton (GVT-0) selfnanoemulsifying drug delivery system (SNEDDS) with myritol as the oil phase, International Journal of Current Innovation Research, 3, 590-594. (2017).

40. M. Yazdanian, K. Briggs, C. Jankovsky, A. Hawi, The "high solubility" definition of the current FDA guidance on biopharmaceutical classification system may be too strict for acidic drugs, Pharm Res, 21, 293-299, (2004).

41. A. Bhatia, P. Shard, D. Chopra, T. Mishra, Chitosan nanoparticles as carrier of immunorestoratory plant extract: synthesis, characterization and immunorestoratory efficacy, International Journal of Drug Delivery, 3,381-385, (2011).

42. R. Martien, B. Loretz, A. Bernkop-Schnürch, Oral gene delivery: design of polymeric carrier systems shielding toward intestinal enzymatic attack, Biopolymers, 83, 327-336, (2006).

43. R. Ravichandran, Nanoparticles in drug delivery: Potential green nanobiomedicine applications, Int J Green Nanotech Biomed, 1, 108-130, (2009).

44. F. Li, J. Li, X. Wen, S. Zhou, X. Tong, P. Su, H. Li, D. Shi, Anti-tumor activity of paclitaxel-loaded chitosan nanoparticles: An in vitro study, Mater Sci Eng C, 29, 2392-2397, (2009).

45. W. F. Tonnis, G. F. Kersten, H. W. Frijlink, W. L. J. Hinrichs, A. H. de Boer, J. P. Amorij, Pulmonary vaccine delivery: a realistic approach?, J Aerosol Med Pulm Drug Deliv, 25, 249-260, (2012).

46. P. Muttil, C. Prego, L. Garcia-Contreras, B. Pulliam, J. K. Fallon, C. Wang, A. J. Hickey, D. Edwards, Immunization of guinea pigs with novel hepatitis B antigen as nanoparticle aggregate powders administered by the pulmonary route, AAPS J, 12, 330-337, (2010). 
47. K. Poelstra, J. Prakash, L. Beljaars, Drug targeting to the diseased liver, J Control Release, 161, 188-197, (2012).

48. R. A. Bader, The Development of Targeted Drug Delivery Systems for Rheumatoid Arthritis Treatment, Rheumatoid Arthritis - Treatment, Dr. Andrew Lemmey (Ed.), ISBN: 978-953-307-850-2, InTech, (2012).

49. M. Higaki, T. Ishihara, N. Izumo, M. Takatsu, Y. Mizushima, Treatment of experimental arthritis with poly(D, L-lactic/glycolic acid) nanoparticles encapsulating betamethasone sodium phosphate, Ann Rheum Dis, 64, 1132-1136, (2005).

50. T. Ishihara, T. Kubota, T. Choi, M. Higaki, Treatment of experimental arthritis with stealth-type polymeric nanoparticles encapsulating betamethasone phosphate, J Pharm Exp Ther, 329, 412-417, (2009).

51. D. Chandrasekar, R. Sistla, F. J. Ahmad, R. K. Khar, P. V. Diwan, The development of folate-PAMAM dendrimer conjugates for targeted delivery of antiarthritic drugs and their pharmacokinetics and biodistribution in arthritic rats, Biomaterials, 28, 504512, (2007).

52. D. Chandrasekar, R. Sistla, F. J. Ahmad, R. K. Khar, P. V. Diwan, Folate coupled poly(ethyleneglycol) conjugates of anionic poly(amidoamine) dendrimer for inflammatory tissue specific drug delivery, $\mathrm{J}$ Biomed Mater Res A, 82, 92-103, (2007).

53. A. S. Chauhan, N. K. Jain, P. V. Diwan, A. J. Khopade, Solubility enhancement of indomethacin with poly(amidoamine) dendrimers and targeting to inflammatory regions of arthritic rats, J Drug Target, 12, 575-583, (2004) 